BNL- 113327-2016-JA

\title{
High-Spectral-Contrast Symmetric Modes in Photonic Crystal Dual Nanobeam Resonators
}

\author{
Siamak Abbaslou, Robert Gatdula, Ming Lu, \\ Aaron Stein, Richard A. Soref, and Wei Jiang
}

Submitted to IEEE Photonics Technology Letters

October 2016

Center for Functional Nanomaterials

Brookhaven National Laboratory

\author{
U.S. Department of Energy \\ USDOE Office of Science (SC), \\ Basic Energy Sciences (SC-22)
}

Notice: This manuscript has been authored by employees of Brookhaven Science Associates, LLC under Contract No. DE- SC0012704 with the U.S. Department of Energy. The publisher by accepting the manuscript for publication acknowledges that the United States Government retains a non-exclusive, paid-up, irrevocable, world-wide license to publish or reproduce the published form of this manuscript, or allow others to do so, for United States Government purposes. 


\section{DISCLAIMER}

This report was prepared as an account of work sponsored by an agency of the United States Government. Neither the United States Government nor any agency thereof, nor any of their employees, nor any of their contractors, subcontractors, or their employees, makes any warranty, express or implied, or assumes any legal liability or responsibility for the accuracy, completeness, or any third party's use or the results of such use of any information, apparatus, product, or process disclosed, or represents that its use would not infringe privately owned rights. Reference herein to any specific commercial product, process, or service by trade name, trademark, manufacturer, or otherwise, does not necessarily constitute or imply its endorsement, recommendation, or favoring by the United States Government or any agency thereof or its contractors or subcontractors. The views and opinions of authors expressed herein do not necessarily state or reflect those of the United States Government or any agency thereof. 


\title{
High-Spectral-Contrast Symmetric Modes in Photonic Crystal Dual Nanobeam Resonators
}

\author{
Siamak Abbaslou, Robert Gatdula, Ming Lu, Aaron Stein, Richard A. Soref, and Wei Jiang
}

\begin{abstract}
We demonstrate accurate control of mode symmetry in suspended dual-nanobeam resonators on a silicon-on-insulator chip. Each nanobeam consists of a Fabry-Perot nanocavity bounded by tapered 1-D photonic crystals. Even and odd cavitymodes are formed due to lateral evanescent coupling between the two nanobeams. The odd cavity-mode can be excited by mode-symmetry-transforming Mach-Zehnder couplers. Modal contrasts over $27 \mathrm{~dB}$ are measured in fabricated structures. The influence of the optical field in the middle air slot on the background transmission and quality factors is discussed. The observed peak wavelength separations of the modes at various nanobeam spacings are in good agreement with simulation results. These nanobeam resonators are potentially useful in applications, such as ultrafast all-optical modulation, filtering, and switching.
\end{abstract}

Index Terms-Photonic crystals, silicon photonics, wavelength filtering devices, coupled resonators, all-optical devices, optical switching devices, guided waves, integrated optics devices.

\section{INTRODUCTION}

$\mathbf{P}$ HOTONIC crystals have opened up abundant opportunities for manipulation of light. Particularly, photoniccrystal nanobeam cavities offer an ideal structure basis for filtering, switching and signal routing in optical interconnect applications owing to their high quality factors, small footprint, compatibility to very large scale integration (VLSI) processing, and scalability [1], [2]. Nanobeams have been incorporated in devices such as photonic-crystal nanobeam lasers [3] and Light Emitting Diodes (LEDs) [4] operating at room temperature, electro-optic modulators with comb-like diode structures [5], [6], high-sensitivity refractive index sensing [7], all-optical switches [8], optomechanics [9], [10], add/drop filters [11] and reconfigurable optical filters [12]. In these devices, nanobeam structures have shown their capability

Manuscript received February 12, 2016; revised May 12, 2016; accepted May 25, 2016. Date of publication June 20, 2016; date of current version August 26, 2016. This work was supported in part by the Defense Advanced Research Projects Agency through the Young Faculty Award under Grant N66001-12-1-4246, in part by the Air Force Office of Scientific Research (AFOSR) under Grant FA9550-08-1-0394, and in part by the U.S. Department of Energy within the Center for Functional Nanomaterials, Brookhaven National Laboratory, Office of Basic Energy Sciences under Grant DE-AC02-98CH10886. The work of R. A. Soref was supported in part by AFOSR under Grant FA9550-14-1-0196 and in part by the U.K. Engineering and Physical Sciences Research Council through the Mid-Infrared Germanium Photonics for Sensing Project. (Corresponding author: Wei Jiang.)

S. Abbaslou, R. Gatdula, and W. Jiang are with the Department of Electrical and Computer Engineering, Rutgers University, Piscataway, NJ 08854 USA (e-mail: s.abaslou@rutgers.edu; rgatdula@rutgers.edu; wjiangnj@rci.rutgers.edu).

M. Lu and A. Stein are with the Center for Functional Nanomaterials, Brookhaven National Laboratory, Upton, NY 11973 USA (e-mail: mlu@bnl.gov; stein@bnl.gov).

R. A. Soref is with the Engineering Department, University of Massachusetts, Boston, MA 02125 USA (e-mail: richard.soref@umb.edu).

Color versions of one or more of the figures in this letter are available online at http://ieeexplore.ieee.org.

Digital Object Identifier 10.1109/LPT.2016.2582641 of achieving high quality (Q) factors, [13] and enhanced light-matter interactions due to their ability to store photons in wavelength-scale volumes for long optical cycles, which also makes them an intriguing platform for cavity quantum electrodynamics [14]. Nanobeam structures have also been shown potential compatibility with complementary metaloxide-semiconductor (CMOS) foundry processes [15].

Dual nanobeams (DNBs) separated by a narrow air-slot cause two cavity modes to strongly inter-couple, generating two modes possessing even and odd symmetry [15]. In all-optical (Kerr) modulation, filtering, and switching applications, accurate control of the mode symmetry and modal wavelength spacings are essential. In principle, symmetry offers selection rules that will allow for unambiguous separation of modes of different symmetry. Experimentally, attaining high-purity even and odd modes has been challenging.

Yet high-purity modes are needed for practical use of nanobeam structures. For example, in ultrafast all-optical modulation [17], filtering [18], [19] and switching [20], a typical scheme may employ a pump laser tuned at one mode (e.g. even mode) to modulate or switch the other mode (e.g. odd mode). An approach to selecting even or odd modes with high purity can be crucial to the device extinction ratio, which is important in all practical applications of optical modulation, switching, and filtering.

In this work, we study the symmetrical modal behavior of photonic-crystal-based dual-nanobeam resonators. Through the use of mode-symmetry-transforming Mach-Zehnder couplers (MZCs), we can excite even, odd, and mixed modes with different configurations of MZCs. To attain a high-purity mode of given symmetry, the optical spectral contrasts against the background transmission and against the mode of opposite symmetry are analyzed. Spectral responses reveal distinctive information about a particular mode.

\section{Principle, Design AND FABrication}

To design the structure, we create in a silicon-oninsulator (SOI) wafer two parallel nanobeams of $450 \mathrm{~nm}$ width, each supporting a single transverse electric (TE) mode around $1550 \mathrm{~nm}$ wavelengths. The Fabry-Perot resonator in each nanobeam consists of two distributed Bragg reflectors (DBRs) or "mirrors". Each DBR comprises a onedimensional photonic crystal having holes arrayed to create a stop band around $1550 \mathrm{~nm}$. The photonic-crystal mirror pitch $\mathrm{a}=360 \mathrm{~nm}$ is linearly tapered over a four hole section to $\mathrm{a}=260 \mathrm{~nm}$ on each side of the nanobeam center to create a resonant transmission mode at around $1550 \mathrm{~nm}$ with a 220 -nm-long cavity at the center. The hole radius is given by $r=0.25 \mathrm{a}$. To attain high-purity modes, we need to design mirrors with high contrast of resonant modes against the background transmission. As the number of holes increases, the Q-factor increases; but it becomes more difficult to couple light into the cavity. To balance the needs for large Q-factor and sufficient transmission of the cavity mode, a moderate 


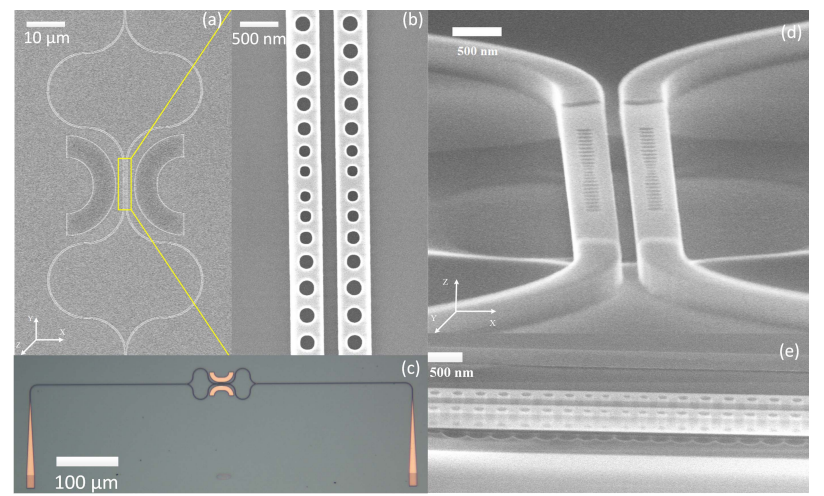

Fig. 1. Scanning electron micrographs (SEM) of (a) DNB resonator with mode-symmetry transforming MZCs, and (b) close-up view of the center part of DNB showing tapered hole array with defects/nanocavities at the center. (c) Optical microscope image of the device showing input/output grating couplers and MZCs. (d) Scanning electron micrograph of suspended DNB. (e) Zoomed side view showing the central portion of the DNB with the etched buried oxide (BOX) layer.

number of 26 holes are employed, 9 mirror holes for each side and 8 tapered holes for the cavity.

The excitation of symmetrical modes with even or odd symmetry is based on the design principles for modesymmetry-transforming MZCs [21]. The scanning electron micrographs (SEM) images of the nanobeams and two MZCs are shown in Fig. 1(a-c). The nanobeams are suspended, as shown in Fig. 1(d-e). Each MZC is a branched Y-junction splitter. We start with a MZC to excite the even mode of the DNB. Here we simply design the arms to have a length difference $(\Delta l)=0$, so there is no phase difference. As for exciting the odd mode, we set the arms to have a length difference $(\Delta l)_{\pi}=\lambda / 2 n_{e f f}$, where $n_{e f f}$ is the effective index of the silicon wire waveguide at $1550 \mathrm{~nm}$. This induces a phase of $\pi$ which excites the odd mode of the DNB. To excite the mixed-mode - a mixture of even and odd modes - we set the length difference $(\Delta l)_{3 \pi / 2}=(3 / 2)(\Delta l)_{\pi}$ which induces a phase difference of $3 \pi / 2$. Numerical simulations with a three-dimensional (3D) finite-difference time-domain method (FDTD) is used to find the mode profile and spectral characteristics of even, odd and mixed modes in the DNB resonator. The simulation results of the DNBs for even and odd modes are shown in Fig. 2(a) and the mixed mode in Fig. 2(b). The different mode profiles for even and odd modes are shown in the inset. Due to the short cavity length $(220 \mathrm{~nm})$, each nanobeam has only one cavity mode that shows no node $(\mathrm{E}=0$ point $)$ in the cavity. Note that the segments of tapered holes are carefully designed to avoid introducing additional resonant modes. The two modes in the two nanobeams interact to generate the even and odd modes. The even mode has a smaller frequency and is the fundamental mode.

The DNB structure is fabricated on a SOI wafer with a $2 \mu \mathrm{m}$ buried oxide layer and a $260 \mathrm{~nm}$ top silicon layer. It is patterned using a JEOL JBX-6300FS high-resolution electron beam (e-beam) lithography system operating at $100 \mathrm{keV}$ on a 120-nm-thick XR-1541-006 hydrogen-silsesquioxane (HSQ) negative e-beam resist. The pattern is transferred to the silicon layer via an Oxford Plasmalab 100 inductive coupled plasma (ICP) etcher, using an $\mathrm{HBr}$ and $\mathrm{Cl}_{2}$ based chemistry for vertical and smooth sidewalls. An etching window to define suspending DNB is patterned via a Karl Suss MJB-3 optical lithography system on $1.4 \mu \mathrm{m}$ of AZ5214E positive photoresist. Through this window, approximately $1 \mu \mathrm{m}$ of

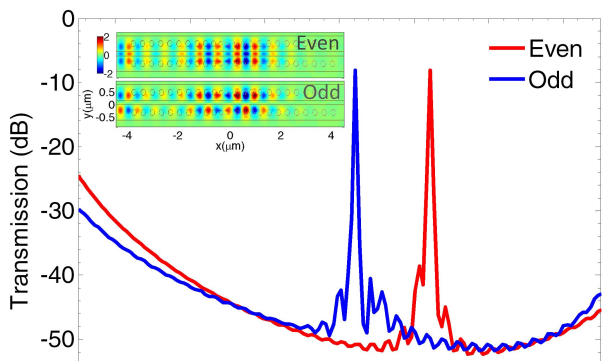

(a)

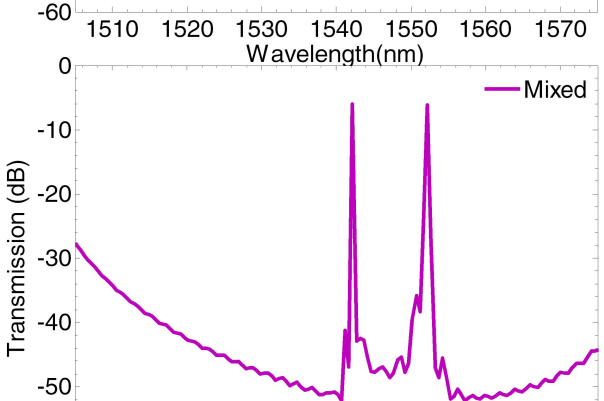

(b)

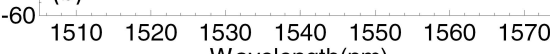
Wavelength $(\mathrm{nm})$

Fig. 2. Numerical simulation results of (a) odd and even modes, and (b) mixed modes for nanobeam spacing $\mathrm{d}=150 \mathrm{~nm}$ and cavity length $\mathrm{D}=220 \mathrm{~nm}$. (inset) The simulated mode profiles for the even and odd modes are shown.

oxide is etched using a buffered oxide etch (BOE) solution. Measurements of the transmission spectra are done by coupling the TE-polarized light from an HP 8168F tunable laser via a single-mode polarization maintaining (PM) fiber array into sub- wavelength grating couplers that deliver the light into the in-plane waveguide structures.

\section{MeAsurements, Results And AnAlysis}

The transmission at each wavelength is recorded via an HP 8153 photo-detector. Fig. 3 shows the spectral responses for even, odd and mixed modes. The spectra are normalized by the transmission spectrum of a reference waveguide with input and output grating couplers. Essential features of the modes agree well with simulation results. In simulations, the wavelength splitting between even and odd mode peaks for the nanobeam spacing $d=150 \mathrm{~nm}$ is $\Delta \lambda=\lambda_{e}-\lambda_{o}=$ $10.03 \mathrm{~nm}$, where $\lambda_{e}=1552.23 \mathrm{~nm}$ and $\lambda_{o}=1542.20 \mathrm{~nm}$, and quality factor $Q_{e} \approx 9.7 \times 10^{3}$ and $Q_{o} \approx 19.0 \times 10^{3}$. In the measured devices, we find $\Delta \lambda=11.2 \mathrm{~nm}$ where $\lambda_{e}=1553.65 \mathrm{~nm}$ and $\lambda_{o}=1542.45 \mathrm{~nm}$, with $Q_{e} \approx 7.6 \times 10^{3}$ and $Q_{o} \approx 12.0 \times 10^{3}$. Based on the inset in Fig. 2 (a), the E-field for the even mode penetrates substantially into the center air gap, which directly connects to the surrounding air and tends to leak light. This may reduce the $\mathrm{Q}$ factor. In contrast, the E-field of the odd mode is confined largely in the silicon nanobeams. As the light tends to stay in higherindex materials, light is less likely to leak to the air and the quality factor is higher. Due to the scattering caused by sidewall roughness for the waveguides and holes as well as light loss from other structure imperfections (e.g. not perfectly vertical sidewalls), the measured Q-factors are lower than the simulation results. The contrast of the selected modes versus the background or undesired modes is rather high. For the even mode excitation given by the red curve in Fig. 3(a), the contrast against the background is about $30.9 \mathrm{~dB}$. For the odd mode 


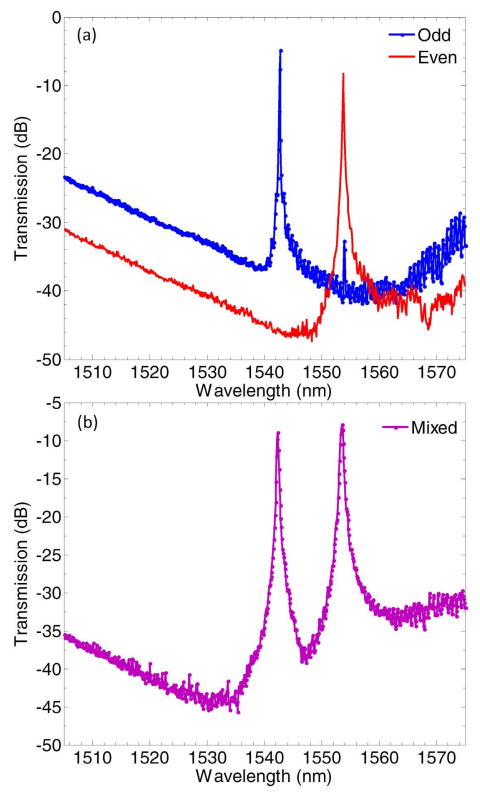

Fig. 3. Experimental measured spectra of (a) odd and even modes, and (b) mixed modes for nanobeam spacing $\mathrm{d}=150 \mathrm{~nm}$ and cavity length $\mathrm{D}=220 \mathrm{~nm}$.

excitation given by the blue curve in Fig. 3(a), the contrast of the odd mode against the background is about $31.2 \mathrm{~dB}$ and over the residual even mode is about $27.8 \mathrm{~dB}$, which is reasonably high compared to photonic-crystal resonators [11] and is also comparable to ring resonators [24].

To analyze the dependence of peak wavelengths of two modes on the nanobeam spacing $d$, we studied the spectral characteristics of mixed-mode excitation with various nanobeam spacings. As shown in Fig. 4, the general trend is that $\Delta \lambda$ decreases as the nanobeam spacing increases. As the nanobeams separate further from each other, their evanescent fields have less coupling, therefore reducing the wavelength splitting due to their coupling. Note that as the spacing $d$ increases, both $\lambda_{e}$ and $\lambda_{o}$ decrease. According to fundamental electromagnetic theory, decrease of the mode wavelength means that the field moves more into the lowindex region (air slot in this case). For the even mode, a substantial portion of the field is in the slot (see Fig. 2(a) inset). As the slot width increases, the portion of the field in the slot increases substantially, so the decreasing trend of $\lambda_{e}$ is fairly strong. For the odd mode, only a very small portion of the field is in the slot because the field must vanish at the slot center line. The decreasing trend of $\lambda_{o}$ is very weak. Also, fabrication processes tend to create small variations of the hole sizes among different structures. Without this non-ideal effect, the peak wavelengths of the modes should vary more smoothly with the spacing $d$. To verify that the fluctuation of the peak wavelengths with $d$ is due to small variations of hole sizes, we have extracted the actual shapes of the holes and nanobeams from SEM micrographs and simulate this actual shape to obtain the simulated peak wavelengths in Fig. 4(b). The simulated results agree fairly well with the experimental results, showing similar fluctuations of the peak wavelengths. There remain small discrepancies between the simulated resonant wavelengths and the measured values in Fig. 4(b). These are attributed to random sidewall roughness.

In Fig. 4(c), the peak wavelength separations obtained from experiments are compared with the peak wavelengths separations of the ideal design (assuming all structures have

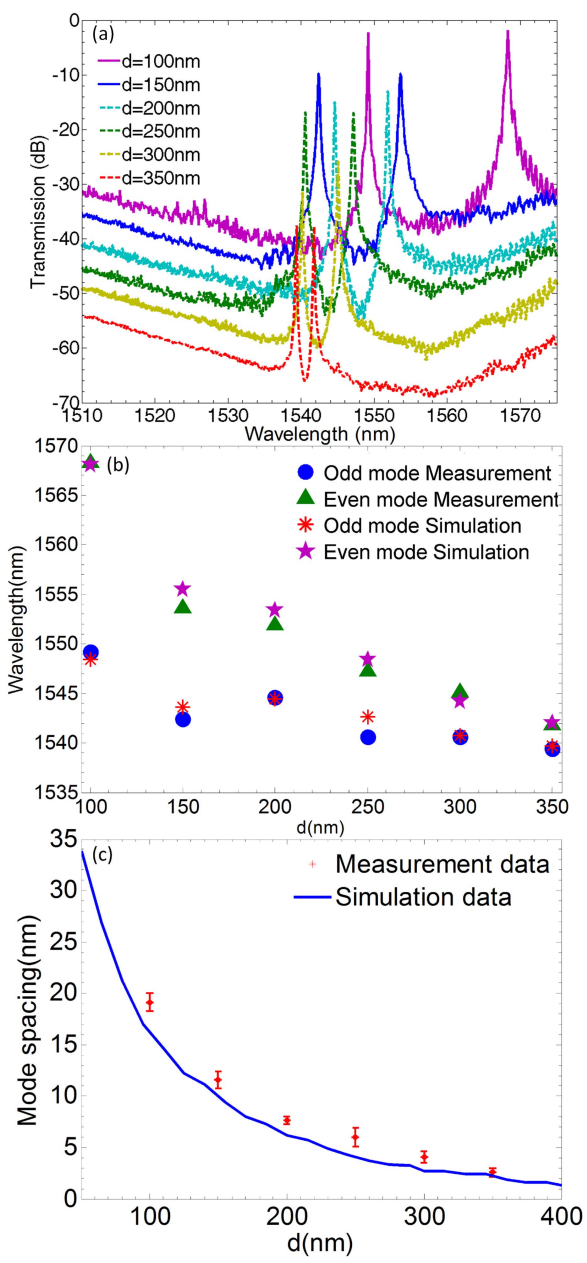

Fig. 4. (a) Measurements of mixed-mode excitations in different slot spacing configurations, showing the trend of bimodal response as the nanobeam spacing between nanobeams increases. For clarity of readings each spectrum offsets by $8 \mathrm{~dB}$. (b) Measurement and simulation results of resonant wavelength for even and odd mode. The structures used in the simulation are extracted from the SEM images of fabricated structures. (c) Mode spacing for different nanobeam spacing. The structures used in the simulations are the ideal design.

the same set of hole sizes but different separations). One finds that although variations of hole sizes in fabricated structures tends to create noticeable fluctuations of the $\lambda_{\mathrm{e}}$ and $\lambda_{\mathrm{o}}$ from the ideal values, the difference $\Delta \lambda$ remains close to the ideal design value. This may be useful in some applications where the device performance depends strongly upon the wavelength separation but is relatively insensitive to small wavelength variation of each mode.

The high contrast of modes against the background and undesired modes obtained here is crucial for practical use of nanobeam devices in all-optical modulation/switching and filtering applications. For example, in a pump-probe configuration where the pump and probe lasers couple to the even and odd modes, respectively (through a mixed-mode MZC at input for example), practical applications often require blocking the relatively high-power pump light and keep the probe signal only at the output. A high-purity odd-mode MZC will be crucial to practical devices in this case. It is possible to further increase the number of holes in the DBR to further suppress the background transmission and increase the quality factor of the cavity. However, as the number of holes increase, scattering loss due 
to small structure imperfection (e.g. roughness) increases, which reduces the peak transmission of the cavity mode. Note that each DBR is essentially a one-dimensional photoniccrystal waveguide. In such a waveguide, the loss is enhanced by the slow light effect [22]. For a specific application scenario, the number of holes in the DBR is determined by the trade-off between the peak transmission and other parameters including the quality factor and background transmission. In fabricated devices, slight asymmetry always exists between two nanobeams and between two arms of the MZC due to structure imperfection (e.g. sidewall roughness) arising from the fabrication processes. This can cause the phases, mode profiles, and other characteristics of two nanobeams or MZC arms to slightly deviate from design. Such deviations are random (causing weak unbalance between two nanobeams) and may distribute over many parts (e.g. over all holes of the nanobeams). This can sometimes cause a very weak residual mode of the opposite symmetry [e.g. in Fig. 3(a)]. The insertion loss of the resonant modes in Fig. 3 can be contributed by the scattering loss due to sidewall roughness of the nanobeam outer edges and the holes, and by the small insertion loss of the MZC. The former is subject to some fluctuation due to the random nature of roughness. Small_fluctuations of input/output grating coupler loss may also slightly affect the loss in Fig. 3. Below 1530nm, the background transmission increases as the wavelength decreases. Generally, as the wavelength $\lambda$ decreases, an optical mode tends to have a large portion of its field moving into the lower-index region. Here, as $\lambda$ decreases, the modes responsible for the background transmission will penetrate more into the slot. As the photonic crystal holes do not extend into the slot, they are less effective to block light in the slot. This contributes to the increase of background transmission at short wavelengths.

Note that as the laser used in our measurements has a low power $(30 \mu \mathrm{W})$, the two-photon absorption and free-carrier generation effects are negligible; therefore, the thermo-optic effect due to free-carrier generation can also be neglected. The low power also reduces any optomechanical effects. In this work, the $Q$-factor refers to the total $Q$ factor, which includes the effect of coupling to the waveguides. The intrinsic $Q_{e}$ has also been previously found to be lower than the intrinsic $Q_{o}$ [15]. Note that the inter-coupling of two nanobeams causes even and odd mode fields to restructure around the slot. The fields at the two outside edges (and outside air-cladding) see very little perturbation due to inter-coupling. The fields there have almost identical magnitudes (opposite sign) for the even and odd modes. Their contributions to the $Q$-factor also have little difference. With careful fabrication, scattering loss due to sidewall roughness is generally lower than the other losses of the resonator, hence has a fairly weak effect on $Q$.

\section{CONCLUSION}

We have demonstrated an approach to precisely control of the mode symmetry in DNB resonator structures utilizing mode-symmetry-transforming MZCs that allow us to excite modes separately into even, odd, or a mixture of the two modes. The difference in the quality factors of the two modes and the characteristics of the background transmission are discussed. Furthermore, we analyzed the bimodal response as it changes with the spacing between the nanobeams. The ability to tune the resonant wavelength of a cavity with mixedmode excitation is particularly interesting for fast low-power all-optical modulation and switching through nonlinear Kerrinduced resonant wavelength shifts (see, for example, [23]).

\section{REFERENCES}

[1] P. B. Deotare, L. C. Kogos, I. Bulu, and M. Lončar, "Photonic crystal nanobeam cavities for tunable filter and router applications," IEEE J. Sel. Topics Quantum Electron., vol. 19, no. 2, Mar./Apr. 2013, Art. no. 3600210

[2] Q. Quan, P. B. Deotare, and M. Loncar, "Photonic crystal nanobeam cavity strongly coupled to the feeding waveguide," Appl. Phys. Lett., no. 96, p. 203102, May 2010.

[3] Y. Gong, B. Ellis, G. Shambat, T. Sarmiento, J. S. Harris, and J. Vučković, "Nanobeam photonic crystal cavity quantum dot laser," Opt. Exp., vol. 18, no. 9, pp. 8781-8789, Apr. 2010.

[4] G. Shambat et al., "Nanobeam photonic crystal cavity light-emitting diodes," Appl. Phys. Lett., no. 99, p. 071105, Aug. 2011.

[5] S. Meister et al., "Matching p-i-n-junctions and optical modes enables fast and ultra-small silicon modulators," Opt. Exp., vol. 21, no. 13, pp. 16210-16221, Jun. 2013.

[6] B. Qi, P. Yu, Y. Li, X. Jiang, M. Yang, and J. Yang, "Analysis of electrooptic modulator with 1-D slotted photonic crystal nanobeam cavity," IEEE Photon. Technol. Lett., vol. 23, no. 14, pp. 992-994, Jul. 15, 2011.

[7] D. Yang, P. Zhang, H. Tian, Y. Ji, and Q. Quan, "Ultrahigh- $Q$ and lowmode-volume parabolic radius-modulated single photonic crystal slot nanobeam cavity for high-sensitivity refractive index sensing," IEEE Photon. J., vol. 7, no. 5, Oct. 2015, Art. no. 4501408

[8] M. Belotti et al., "All-optical switching in silicon-on-insulator photonic wire nano-cavities," Opt. Exp., vol. 18, no. 2, pp. 1450-1461, Jan. 2010

[9] M. Li, W. H. P. Pernice, and H. X. Tang, "Tunable bipolar optical interactions between guided lightwaves," Nature Photon., vol. 3, pp. 464-468, Jul. 2009.

[10] M. Eichenfield, R. Camacho, J. Chan, K. J. Vahala, and O. Painter, "A picogram- and nanometre-scale photonic-crystal optomechanical cavity," Nature, vol. 459, pp. 550-555, May 2009.

[11] C. V. Poulton, X. Zeng, M. T. Wade, and M. A. Popović, "Channel adddrop filter based on dual photonic crystal cavities in push-pull mode," Opt. Lett., vol. 40, pp. 4206-4209, Aug. 2015.

[12] P. B. Deotare et al., "All optical reconfiguration of optomechanical filters," Nature Commun., vol. 3, May 2012, Art. no. 846.

[13] I. W. Frank, P. B. Deotare, M. W. McCutcheon, and M. Lončar, "Programmable photonic crystal nanobeam cavities," Opt. Exp., vol. 18, no. 8, pp. 8705-8712, Apr. 2010.

[14] A. Rundquist, A. Majumdar, and J. Vučković, "Off-resonant coupling between a single quantum dot and a nanobeam photonic crystal cavity," Appl. Phys. Lett., vol. 99, no. 25, p. 251907, Dec. 2011.

[15] P. B. Deotare, M. W. McCutcheon, I. W. Frank, M. Khan, and M. Lončar, "Coupled photonic crystal nanobeam cavities," Appl. Phys. Lett., vol. 95, no. 3, p. 031102, Jul. 2009.

[16] C. V. Poulton, X. Zeng, M. T. Wade, J. M. Shainline, J. S. Orcutt, and M. A. Popović, "Photonic crystal microcavities in a microelectronics 45-nm SOI CMOS technology," IEEE Photon. Technol. Lett., vol. 27, no. 6, pp. 665-668, Mar. 15, 2015.

[17] J. Hendrickson, R. Soref, J. Sweet, and W. Buchwald, "Ultrasensitive silicon photonic-crystal nanobeam electro-optical modulator: Design and simulation," Opt. Exp., vol. 22, no. 3, pp. 3271-3283, Feb. 2014.

[18] X. Ge, Y. Shi, and S. He, "Ultra-compact channel drop filter based on photonic crystal nanobeam cavities utilizing a resonant tunneling effect," Opt. Lett., vol. 39, no. 24, pp. 6973-6976, Dec. 2014.

[19] W. S. Fegadolli, J. E. B. Oliveira, V. R. Almeida, and A. Scherer, "Compact and low power consumption tunable photonic crystal nanobeam cavity," Opt. Exp., vol. 21, no. 3, pp. 3861-3871, Feb. 2013.

[20] N. Vukovic et al., "Ultrafast optical control using the Kerr nonlinearity in hydrogenated amorphous silicon microcylindrical resonators," Sci. Rep., vol. 3, Oct. 2013, Art. no. 2885

[21] J. Tan, M. Lu, A. Stein, and W. Jiang, "High-purity transmission of a slow light odd mode in a photonic crystal waveguide," Opt. Lett., vol. 37, no. 15 , pp. 3189-3191, Jun. 2012.

[22] W. Song, R. A. Integlia, and W. Jiang, "Slow light loss due to roughness in photonic crystal waveguides: An analytic approach," Phys. Rev. B, vol. 82, no. 23, p. 235306, Dec. 2010.

[23] G.-R. Lin et al., "Si-rich $\mathrm{SiN}_{\mathrm{X}}$ based Kerr switch enables optical data conversion up to 12 Gbit/s," Sci. Rep., vol. 5, Apr. 2015, Art. no. 9611.

[24] W. Bogaerts et al., "Compact wavelength-selective functions in siliconon-insulator photonic wires," IEEE J. Sel. Topics Quantum Electron., vol. 12, no. 6, pp. 1394-1401, Nov. 2006. 